\section{Self-regulation and medical direction}

Self-regulation and medical direction

\section{Conflicted approaches to monitoring and improving the quality of clinical care in paramedic services}

\author{
Peter O’Meara \\ School of Rural Health, La Trobe University - Bendigo Campus, \\ Bendigo, Australia \\ Gary Wingrove \\ Mayo Clinic Medical Transport, Rochester, Minnesota, USA and \\ The Paramedic Foundation, St Cloud, Minnesota, USA, and \\ Michael McKeage \\ Bruce County Health Services, Walkerton, Canada
}

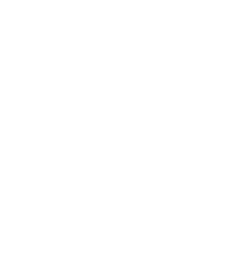

\begin{abstract}
Purpose - The purpose of this paper is to describe and analyse two approaches to paramedic service clinical governance and quality management from the perspective of two groups of paramedics and paramedic managers working in North America.

Design/methodology/approach - A case study approach was utilised to describe and analyse paramedic service medical direction in North America and contrast this with the professional self-governance and clinical governance systems operating in other high-income countries. Researchers interviewed participants at two remote North American sites, then completed transcription and thematic analysis.

Findings - Participants identified three themes: first, resourcing, regulatory frameworks and fragmentation; second, independent practice facilitators and barriers; and third, paramedic roles and professionalisation. Those trained outside North America tended to identify self-regulation and clinical governance as the preferred approach to quality management. Few participants had considered paramedicine becoming a self-regulating health profession.

Originality/value - In North America, the "medical direction" model is the dominant approach employed to ensure optimal patient outcomes in paramedic service delivery. In contrast, other comparable countries employ paramedic self-regulatory systems combined with clinical governance to achieve the same ends. This is one of two studies to examine medical direction from the perspective of paramedics and paramedic managers.
\end{abstract}

Keywords Clinical governance, Quality, Qualitative research, Governance structures, Organizational development for effective clinical governance, Clinical leadership and culture

Paper type Research paper

\section{Introduction}

Paramedic services are organised, funded and managed in a variety of ways, depending on their cultural and health system context. They are generally divided into two main models: the Franco-German model that is predominately physician-staffed; and the Anglo-American model that uses paramedics, with a variety of role titles, to staff ambulances as a distinct

(C) Peter O'Meara, Gary Wingrove and Michael McKeage. Published by Emerald Publishing Limited. This article is published under the Creative Commons Attribution (CC BY 4.0) licence. Anyone may reproduce, distribute, translate and create derivative works of this article (for both commercial \& non-commercial purposes), subject to full attribution to the original publication and authors. The full terms of this licence may be seen at http://creativecommons.org/licences/by/4.0/legalcode. Thanks to La Trobe University for funding the Chief Investigator's travel. In-kind support was provided by the study sites.

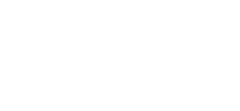


IJHG

23,3

234

occupational group (Timmermanna et al., 2008). The latter model is dominant in high-income countries such as the USA, Canada, Ireland, UK, Australia and New Zealand.

On closer examination of the Anglo-American model, notable differences in how these paramedic services operate are evident. One difference is the approach used to monitor and improve the quality and safety of clinical services. In North America, the "medical direction" model is the dominant approach employed to ensure optimal patient outcomes in paramedic service delivery (National Association of EMS Physicians, 2010), while in the UK, Ireland, Australia and New Zealand professional self-regulation combined with clinical governance frameworks is the preferred approach for all health services, including paramedic services (Safer Care Victoria, 2017).

There is little research comparing these contrasting quality management approaches. Nor did we find in our review of the literature any strong evidence supporting the effectiveness of either approach in paramedic services (O'Meara et al., 2017). For paramedics operating in systems where they are recognised and practice as autonomous health practitioners the medical direction model is perplexing (Council of Ambulance Authorities, 2014), while others aspiring to self-regulation find the concept frustrating (Ontario Paramedic Association, 1991/2013). One Canadian conference paper used rhetorical genre theory to challenge medical direction on the grounds that it is a form of continuing medical dominance over the work of paramedics (Fitzgerald, 2014). More recently, a survey of front-line paramedics in Ontario, Canada found mixed perceptions about medical direction in their unique "base hospital" system (Foerster et al., 2018).

These observations indicate that a better understanding of the medical direction model is required before undertaking direct comparisons of medical direction with self-regulatory and clinical governance systems. While there are numerous publications related to medical direction, they are invariably descriptive, non-critical, lack evidence and do not consider alternate models (O'Meara et al., 2017; Millin et al., 2011). With the exception of the Ontario study, the perspectives of paramedics trained and/or practicing in North America about medical direction or alternate quality management systems are largely unknown.

The purpose of this study was to describe and analyse paramedic service clinical governance and quality improvement systems in North America from the perspective of paramedics and paramedic managers as expert informants. The specific aims were to:

(1) describe and compare the essential elements of medical direction and clinical governance (if possible) in paramedic services;

(2) identify the strengths and weakness of medical direction (and if possible selfregulation and clinical governance) in the paramedicine context; and

(3) consider the implications of the findings, and determine how each of these approaches to quality assurance can inform the other.

\section{Methods}

A case study approach, inspired by Yin (2003), was used to describe and analyse the operation of medical direction in North America, and where possible to contrast this with self-regulatory and clinical governance frameworks used in other high-income countries (World Bank, 2017). Using case studies offered an opportunity to examine the roles and accountabilities of paramedics, physicians and managers in remote and relatively under-regulated paramedic services in countries' respective paramedicine systems within the Anglo-American model of service delivery.

In-depth interviews with practicing paramedics and paramedic service managers were audio-recorded at two remote sites in North America and later transcribed for thematic analysis (Braun and Clarke, 2006). The selection of these study sites and participants enhanced the 
value and richness of the findings of how paramedic service clinical quality monitoring and assurance programs operate in remote locations of North America where there are great variations in staffing and governance models (National Highway Traffic Safety Administration, 2014). Two of the investigators drafted and refined the interview questions to match their relevance to the research question and to ensure that participants would understand the terminology. For example, asking direct questions about clinical governance was rejected on the basis that paramedics in the USA would probably be unfamiliar with the term, while direct questions about medical direction could have been viewed as "leading questions". One of the investigators and an independent research assistant integrated and analysed the interview transcripts using classic thematic analysis techniques. The chief investigator drafted the manuscript with the local investigators then contributing.

\section{Organisational settings, participants and data collection}

The researchers purposively selected two remote sites in Canada and the USA on the basis of their remoteness and relatively under-developed governance systems, as well as the likelihood that potential participants had been exposed to volunteer providers. This is important as there are more than 21,000 Emergency Medical Service (EMS) agencies in the USA, with volunteer providers playing an integral role in providing prehospital care in one-third of cases (National Highway Traffic Safety Administration, 2014). Both these paramedic services recruit paramedics from throughout North America, and in some cases from the UK and Australia where self-regulation and clinical governance systems are more established.

The Canadian paramedic service was an established, government-funded paramedic service operating in a remote location. The US paramedic service was a hospital-based provider that in this case was servicing a large transient community attending an extended cultural event in a remote location over a period of approximately two weeks, with additional personnel temporarily engaged from other parts of the USA and internationally for this short-duration event.

The La Trobe University Human Research Ethics Committee gave approval for the study. The Territorial Government, through a Scientists and Explorers Act, gave approval for the Canadian arm of the study. The US site accepted the University Human Research Ethics Committee approval.

Local investigators invited paramedics and paramedic managers to participate. The interviewing investigators explained the study to each participant in detail, obtained consent and then conducted the interviews in private settings. The study was anonymous with no identifying names, marks or codes on any data that was analysed in accordance within the academic conventions that respect participants' identities and the confidentiality of their data. In total, 16 paramedics and paramedic managers were interviewed - seven from the Canadian site and nine from the US site. Interview duration varied from 9 to $46 \mathrm{~min}$, with most taking approximately $20 \mathrm{~min}$.

These paramedics and paramedic managers gave their perspectives on paramedic services' quality management approaches. As expert informants qualified to make meaningful and informed contributions, they described the quality management system that operates in their paramedic services, and outlined its essential elements, strengths and weakness. In order to provide some sense of the perspective from which they were answering the questions they described their role within their local clinical quality monitoring and assurance system.

\section{Findings}

Participants raised a wide range of issues related to quality management, with the more senior and experienced providing more information and greater insight. The difficulty that recently graduated participants had answering the interview questions, made it evident that quality management is not included in the formal education and training of 
IJHG

23,3

paramedics in North America. The more seasoned participants reported that they had developed their knowledge of quality management, regulation and governance through experience.

Those who had trained and worked in the North America generally saw medical directors leading and implementing these processes. In contrast, those from the UK and Australia identified clinical governance as their preferred approach. A small number of participants who had trained in the UK, Australia and some parts of Canada identified self-regulation as a way forward. They did not explain how this would be implemented within clinical governance systems where best practice suggests that there should be a division between governance, management and practice roles (Brennan and Flynn, 2013).

The participants identified three inter-related themes clustered around:

(1) resourcing, fragmentation and regulatory frameworks;

(2) independent practice facilitators and barriers; and

(3) paramedic roles and professionalisation.

\section{Resourcing, fragmentation and regulatory frameworks}

The strongest theme that emerged from the interviews was the incredible diversity and fragmentation that is apparent in North America, particularly in the US where "paramedic services" have widely varying funding sources, organisational structures, staffing and regulatory regimes (National Highway Traffic Safety Administration, 2014). While there was a sense that paramedic services and managers had the best of intentions, the necessary resources were limited:

The management team is overwhelmed with a high volume of work and a lot of deliverables within an ineffective support staff compliment [...] there's a huge disconnect on this. Which is not because of the individuals and it's not because of a lack of desire to work as a cohesive team, there's just not enough hours in the day. (Participant 2)

Because of limited resources, the extent of quality monitoring and improvement activities was often inconsistent across agencies, or in some cases almost invisible:

We do have certain things that we have to do for quality assurance, but I don't believe that there is a solid thing that everybody is doing. [...] you can see the differences in the standard of care from one jurisdiction to another. (Participant 10)

I do know that EMS did talk QA a number of years ago, I guess back in that moment of time it was important. I think they really did want to give it attention. Whether budgets, whether personnel, whatever happened, I haven't heard those terms mentioned in a number of years now. (Participant 7)

Smaller agencies often relied upon "personality driven" quality management systems, with the medical director considered the key to action. In contrast, those operating in larger systems had greater access to the resources and infrastructure required to implement and maintain effective and sustainable quality management systems:

It's a small, small organisation so we have face to face contact with the people that are making these overall decisions, so I can email the medical director when we have one and make suggestions and directly get feedback almost immediately via email or face to face conversations [...] so there is personal sort of affect there as well. (Participant 4)

We have an Incident Information Management System, a little bit complicated but it's not that hard to go through, probably takes about twenty minutes if it's something complicated, we report all of the incidents through that. (Participant 12) 
This lack of adequate resourcing was described as severe in rural and frontier settings across both the USA and Canada where paramedic services are often voluntary, and lack the resources to provide more than basic services, leaving few resources for management infrastructure and quality assurance (National Highway Traffic Safety Administration, 2014; Freeman et al., 2008). The participants reported that volunteers "push back" against additional management processes and inflexible regulatory requirements in rural and frontier areas:

The other issue that we had is we're a very rural area and in [State], eighty five percent of EMS is provided by volunteers and those volunteers work full-time jobs and are called to respond either as a first responder or a transport agency depending on the state. (Participant 11)

One of the challenges in the USA is a fragmented system design where there are marketedly different models of service provision and organisational structures found throughout the country (National Highway Traffic Safety Administration, 2014), with each region having the ability to set its own clinical and credentialing standards, and clinical protocols. In some Canadian provinces, including Ontario, this pattern can also be seen (Ministry of Health and Long-Term Care, Emergency Services Branch, 2018):

We have a service with three different medical control areas that each have their own independent medical protocols, their own independent medical oversight and credentialing process, and their own Independent quality review processes. (Participant 14)

I think that having one medical director creates a subjective bias that changes the whole view over the service and that is one of the things that I think has created a huge disparity between EMS services across the United States. (Participant 11)

There were a number of accounts of states across the USA having multiple medical directors and quality management systems. It was reported that there are "[...] 46 medical directors in the state and the opportunity for forty six different systems" (Participant 11), while in another state there were 38 systems, each with a medical director, an administrator, a trauma coordinator, a communications person, a logistics kind of person to manage the systems (Participant 15). These types of medical director governance models can allow arbitrary application of clinical protocols and certification standards; potentially preventing paramedics certified in one paramedic service from concurrently practicing at the same scope of practice in an adjoining service.

This is in marked contrast to paramedic systems in other high-income countries. For instance in England, there are ten Ambulance Service NHS Trusts that operate under national clinical practice guidelines that:

[...] are an important part of clinical risk management and ensure uniformity in the delivery of high quality patient care. As such they form the basis for UK paramedic training and education [...] The guidelines are reviewed on a five year cycle and are developed or updated, based on systematic reviews of the evidence and consensus agreement. (Joint Royal Colleges Ambulance Liaison Committee Guideline Development Group, 2017)

All paramedics in the UK are nationally registered health professionals under the Health and Care Professionals Council (Woollard, 2009; Association of Ambulance Chief Executives, 2017). This regulatory regime contrasts with the systems in the US where paramedics are unable to function unless under the delegated medical practice of a physician medical director. Medical directors in the USA are responsible for patient care activities performed by paramedics, taking responsibility for their appropriateness, and that these activities are within their scope of practice and operational expectations (Freeman et al., 2008; Garza et al., 2012; Bass et al., 2015). One US participant made the point that in their paramedic service; their continuing capacity to practice and remain employed is dependent on re-credentialing by the medical director (Participant 11). Medical direction also
Self-regulation and medical direction 
IJHG

23,3

238

involves the all-encompassing roles of signing off on clinical protocols and implementing best practice. Despite these concerns, approximately half of the participants seemed happy to retain the medical oversight model because of the wide variety of training and continuing education that paramedics receive across the country:

I think it's important to work in partnership with physicians because I think physicians do have that twelve years of education and they have a little bit more knowledge of what their practice is with the EMS provider, but I think we're partners in that healthcare system. (Participant 4)

\section{Independent practice facilitators and barriers}

The possibility of independent paramedic practice being established and sustained is dependent on the existence of a combination of adequate resourcing, underpinned by strong infrastructure in the form of a robust and well-rounded education system, and a strong professional identity amongst paramedics that is manifested through the establishment of professional colleges or similar bodies (Lyndon-James, 2013; Sheather, 2009). It was observed that in the USA paramedic education was often under the control of medical directors, rather than paramedics through professional colleges:

The way that it's set up is that the National Curriculum is there as a guideline and each State has the option to adopt that curriculum in its entirety as their basis or they can alter it, and in our State it was altered according to the medical director. (Participant 11)

These professional underpinnings vary and are not always be in step with each other. One perspective from participants was that medical direction provides a level of safety because paramedics in the USA are not clinicians in their own right, while others felt that Clinical Practice Guidelines would allow paramedic practitioners to practice the best quality of care, rather than be dependent on medical director oversight. The same participant made the point that:

The education system (paramedic) has evolved over the years; however, the power of the doctor has not. (Participant 2)

Up to half of the participants saw little need or prospect of changing from medical direction, even when they were criticising this model for being ineffective and sometimes punitive. These views are consistent with similar findings in a Canadian study of the professional relationships between paramedics and medical directors, where respondents identified an association between medical direction and a punitive culture that discouraged paramedic critical thinking (Foerster et al., 2018). Although the National Association of EMS Physicians offers training and certification for medical directors, this is not universal throughout North America, and any licensed physician can fill the role in a number of states and provinces (Bass et al., 2015; National Association of Emergency Medical Service Physicians, 2016). Limited numbers of medical directors have completed any certification designed to ensure they are competent and current in their provision of medical oversight duties. It was clear that in the systems described the medical director role is both fundamental and flawed:

Self-governance, you know I think we're treading on thin ice there. You know the doctors are the trained professionals and we're like one of their hands or we're their eyes or something. We don't have all of the stuff that we need for self-governance and I think that a doctor is always going to need to be there, a medical director, somebody who can see what's needed [...] to fit the exact situation. (Participant 9)

The medical director will strongly set the tone and the culture of the organisation [...] I believe that even with the new certification that medical directors can get, I do not believe that there is a set standard for how they run their services. (Participant 11) 
The establishment of a strong professional identity, manifested through the establishment of professional colleges or similar bodies is a precursor to paramedics embracing self-regulation and forms of clinical governance as a means of assuring the quality and safety of the services and care they provide to the community (McCann et al., 2013). While paramedic systems that have made strides towards self-regulation and clinical governance continue to value the input of physicians, there is a marked difference in the power dynamic between paramedics, regulatory bodies and medical advisors:

[...] we're under a board, a medical advisory board which has regional doctors and city doctors as well as paramedic representation and community representation that sort of oversees or at least looks at all of those other things as well. (Participant 12)

Participants noted that there is considerable deference given to physicians that bordered on "doctor worship" borne of an apparent professional inferiority complex that might be explained as a result of having gone through a paramedic education system based on skill acquisition and rote learning rather than critical thinking and independent decision making. There were strong calls from participants for improved paramedic education that would lead to better regulatory systems and a stronger knowledge base for the profession:

If I was going to create a new system [...] I'd make sure that everybody went through a college education or a professional education, that they were licensed not certified, licensed at a minimal level same as nurses, same as physicians. (Participant 11)

I can tell you both personally, professionally and from an advocacy role I would like to see self- regulation in [Province], I would like to see a College of Paramedics that works primarily hand in hand with the Provincial Health Services Authority for clinical governance, quality assurance. (Participant 8)

One US participant was critical of potential conflicts of interest in health systems that provide perverse incentives to take patients to emergency departments and hospitals even when there is no clinical need:

That Medical Advisory Board [is] [...] pretty much dominated by the doctors from the local hospitals and it seems to me that the profitability of those hospitals was paramount in their minds as far as regulations. [...] there's some undue influence that's brought about from some of the hospitals that are more based towards profit and not on good patient care. (Participant 9)

Perhaps because of uncertainty about paramedics' willingness to professionalise, some participants singled out for criticism those physicians who had been paramedics:

I get concerned about a lot of doctors who used to be paramedics who think that they're just like the paramedic on steroids now because they're a doctor. They really have no current understanding of paramedicine and what it takes, because it's more than just the skills, it's everything that goes with the package that provides the skills. But they still think of it as skills. [...] There are people out there that people [paramedics] practically worship [...] it just blows my mind because they have done nothing to progress our profession, if they had we wouldn't be in the position we are right now. (Participant 15)

\section{Paramedic roles and professionalization}

Participants were keen to argue that change was required, and they could see new roles emerging such as community paramedics. However, the prospects for self-regulation were mixed, with some optimistic, while others expressed the view that paramedicine lacked the maturity to take this step towards autonomous practice. Some were critical of existing professional associations that lack the traction to change the status quo. 
IJHG

23,3

240

At an individual level, the participants expressed aspirations to improve the paramedic system and their own paramedic services in particular. Some felt that self-regulation and professional pride were linked concepts:

In the ideal world in the U.S., paramedics would take personal responsibility and accountability for everything that they did clinically or otherwise and would always be looking for opportunities for improvement of themselves, so by improving one-self you raise the whole system up. (Participant 15)

However, broader knowledge and understanding of how self-regulation and clinical governance could have an impact on quality and safety was scant amongst those participants without exposure to paramedic regulation and clinical governance in other countries. There was a cry for better monitoring and improvement systems that would make a difference to paramedic practice and improve patient outcomes. However, there was limited understanding of the distinction between governance, management and practice with the result that participants often saw little alternative to the all-encompassing medical director role where these responsibilities are often blurred. This fall back to embracing "personality-driven systems of quality management", even when effective, seems to stand in the way of consistent and sustainable improvement in paramedic practice, greater levels of professionalisation and self-regulation.

\section{Discussion}

The participants in this study each had their own vision for the future that varied from maintaining the status quo to the professionalisation of paramedicine through research and education, professional self-regulation and the development of a paramedicine professional identity. In the USA, in particular, progressing these aspirations will rely on a reduction in organisational and professional fragmentation, and the removal of legislative and regulatory impediments designed before current paramedic capabilities and professional practice standards were in place.

Medical direction has a strong hold over paramedicine in North America, where paramedics and managers hold little power to change a system that is embedded in legal structures and accreditation requirements (Bass et al., 2015; Wydro et al., 1997). This contrasts with the situation in other high-income countries operating within the Anglo-American paramedicine system, where professional self-regulatory boards and clinical governance support the growth of an autonomous paramedicine profession (O'Meara et al., 2017; Wankhade and Mackeay-Jone, 2015).

While paramedic roles and scopes of practice are expanding in both depth and breadth, fragmentation and inadequate resourcing for paramedicine makes any system changes challenging (Freeman et al., 2008). In Canada, where there is less fragmentation and strong paramedic leadership, the push towards self-regulation is advancing in a number of provinces despite opposition from some stakeholders and limited resources (Fitzgerald, 2014; Toews, 2017). The long-term prospect of building an autonomous paramedicine profession through self-regulation and clinical governance depends on creating a paramedic professional identity, underpinned by paramedic research and education that matches independent paramedic practitioner roles and scopes of practice. Other emerging health professions have travelled this path towards professional recognition and paramedicine can draw some optimism from their ability to define and negotiate their own practice and conduct standards (Allan, 1997).

\section{References}

Allan, D. (1997), "The nursing-medical boundary: a negotiated order?", Sociology of Health and Illness, Vol. 19 No. 4, pp. 498-520.

Association of Ambulance Chief Executives (2017), "Structure of the UK ambulance services", available at: https://aace.org.uk/uk-ambulance-service/ (accessed 15 August 2017). 
Bass, R.R., Lawner, B., Lee, D. and Nable, J.V. (2015), "Medical oversight of EMS systems", in Cone, D.C., Brice, J.H., Delbridge, T.R. and Myers, B. (Eds), Emergency Medical Services: Clinical Practice and Systems Oversight, Vol. 2, John Wiley \& Sons, Chitchester, pp. 71-83.

Braun, V. and Clarke, V. (2006), "Using thematic analysis in psychology", Qualitative Research in Psychology, Vol. 3 No. 2, pp. 77-101.

Brennan, N.M. and Flynn, M.A. (2013), "Differentiating clinical governance, clinical management and clinical practice", Clinical Governance: An International Journal, Vol. 18 No. 2, pp. 114-131.

Self-regulation and medical direction

Council of Ambulance Authorities (2014), Guidelines for the Assessment and Accreditation of Entry-level Paramedic Education Programs, Council of Ambulance Authorities and Paramedics Australasia, Melbourne.

Fitzgerald, S. (2014), "More than just ambulance drivers: a genre struggle for professional recognition", 6th Annual Conference of the Canadian Association for the Study of Discourse and Writing, Brock University, St Catharines, 24-26 May.

Foerster, C., Tavares, W., Virkkunen, I. and Kämäräinen, A. (2018), “A survey of front-line paramedics examining the professional relationship between paramedics and physician medical oversight", Canadian Journal of Emergency Medicine, Vol. 20 No. 2, pp. 238-246.

Freeman, V., Patterson, D. and Slifkin, R. (2008), "Issues in staffing emergency medical services: a national survey of local rural and urban EMS directors", Final Report No. 93, North Carolina Rural Health Research and Policy Analysis Center, Grand Forks.

Garza, A. and Mitchell, E. (2012), in FEMA (Ed.), Handbook for EMS Medical Directors, US Department of Homeland Security, Washington, DC, p. 92.

Joint Royal Colleges Ambulance Liaison Committee Guideline Development Group (2017), "The JRCALC clinical practice guidelines", available at: www2.warwick.ac.uk/fac/med/research/ hsri/emergencycare/prehospitalcare/jrcalcstakeholderwebsite/ (accessed 15 August 2017).

Lyndon-James, P. (2013), "Striving for the professionalisation of Australian paramedics”, International Paramedic Practice, Vol. 3 No. 3, pp. 74-78.

McCann, L., Granter, E., Hyde, P. and Hassard, J. (2013), "Still blue-collar after all these Years? An ethnography of the professionalization of emergency ambulance work", Journal of Management Studies, Vol. 50 No. 5, pp. 750-776.

Millin, M.G., Brown, L.H., Craven, C.K., Hawkins, S.C., Tan, D.K., Piazza, G.M., Cone, D.C. and Sattin, R.W . (2011), "Evolution of the literature identifying physicians' roles in leadership, clinical development, and practice of the subspecialty of emergency medical services", Prehospital and Disaster Medicine, Vol. 26 No. 1, pp. 49-64.

Ministry of Health and Long-Term Care (2018), Emergency Services Branch: Statistics, available at: www.health.gov.on.ca/english/public/program/ehs/ehs_dt.html (accessed 23 January 2018).

National Association of EMS Physicians (2010), "National association of state EMS officials. Medical direction for operational emergency medical services programs", Prehospital Emergency Care, Vol. 14 No. 4, pp. 544-544.

National Association of Emergency Medical Service Physicians (2016), "Innovations in EMS fellow education", Prehospital Emergency Care, Vol. 20 No. 2, pp. 307-309.

National Highway Traffic Safety Administration (2014), "EMS System Demographics”, US Department of Transportation, Washington, DC.

O’Meara, P., Wingrove, G. and Nolan, M. (2017), "Clinical leadership in paramedic services: a narrative synthesis", International Journal of Health Governance, Vol. 22 No. 4, pp. 251-268.

Ontario Paramedic Association (1991/2013), "Application for regulation of paramedics under the regulated health professions act.

Safer Care Victoria (2017), Delivering High-Quality Healthcare: Victorian Clinical Governance Framework, Victoria State Government, Melbourne.

Sheather, R. (2009), "Professionalisation", in O'Meara, P. and Grbich, C. (Eds), Paramedics in Australia: Contemporary Challenges of Practice, 1st ed., Pearson Education Australia, Sydney, pp. 62-83. 
IJHG

23,3

242

Timmermanna, A., Russoa, S.G. and Hollmann, M.W. (2008), "Paramedic versus emergency physician emergency medical service: role of the anaesthesiologist and the European versus the Anglo-American concept", Current Opinion in Anesthesiology, Vol. 21 No. 2, pp. 222-227.

Toews, R. (2017), Consultant Report on the Implementation of Self-regulation for Paramedics, Ministry of Health, Seniors and Active Living, Winnipeg.

Wankhade, P. and Mackeay-Jone, K. (2015), Ambulance Services: Leadership and Management Perspectives, Springer, Switzerland.

Woollard, M. (2009), "Professionalism in UK paramedic practice", Journal of Emergency Primary Health Care, Vol. 7 No. 4.

World Bank (2017), "How does the World Bank classify countries?", available at: https://datahelpdesk. worldbank.org/knowledgebase/articles/378834-how-does-the-world-bank-classify-countries (accessed 15 December 2017).

Wydro, G.C., Cone, D.C. and Davidson, S.J. (1997), "Legislative and regulatory description of EMS medical direction: a survey of states", Prehospital Emergency Care, Vol. 1 No. 4, pp. 233-237.

Yin, R. (2003), Application of Case Study Research, SAGE Publications, London.

\section{Corresponding author}

Peter O’Meara can be contacted at: p.omeara@latrobe.edu.au

For instructions on how to order reprints of this article, please visit our website: 